\title{
Bayesian inference and Markov chain Monte Carlo based estimation of critical slip distance parameter in rate and state model for fault friction
}

\author{
Saumik Dana \\ University of Southern California \\ Los Angeles, CA 90007 \\ sdana@usc . edu
}

\begin{abstract}
We present an algorithmic framework to solve an inverse problem using Bayesian inference and Markov chain Monte Carlo sampling. The input of the inverse problem is the acceleration of the slipping seismogenic fault and the output is the probability distribution of the critical slip distance parameter of the rate and state model for fault friction.
\end{abstract}

\section{Introduction}

Earthquakes are triggered after critically stressed seismogenic faults slip [1] after being activated due to natural causes like earth tides [2], rainfall [3], snowfall [4], typhoons [5], as well as human activity [6]. The component linking fault activation and fault slip is the popularly used rate- and state- dependent friction (RSF) model for friction evolution [7-9] given by

$$
\begin{gathered}
\mu=\mu_{0}+A \ln \left(\frac{V}{V_{0}}\right)+B \ln \left(\frac{V_{0} \theta}{d_{c}}\right) \\
\dot{\theta}=1-\frac{\theta V}{d_{c}}
\end{gathered}
$$

where $V=|d \boldsymbol{d} / d t|$ is the slip rate magnitude, $\mu_{0}$ is the steady-state friction coefficient at the reference slip rate $V_{0}, A$ and $B$ are empirical dimensionless constants, $\theta$ is the macroscopic variable characterizing state of the surface and finally $d_{c}$ is a critical slip distance. The critical slip distance is the distance over which a fault loses or regains its frictional strength after a perturbation in the loading condi- 
tions [10]. In principle, it determines the maximum slip acceleration and radiated energy to such an extent that it influences the magnitude and time scale of the associated stress breakdown process [11]. Regardless of the importance, it is paradoxical that the values of $d_{c}$ reported in the literature range from a few to tens of microns as determined in typical laboratory experiments [11], to $0.1-5 \mathrm{~m}$ as determined in numerical and seismological estimates based on geophysical observations [12], and further to several meters as determined in high-velocity laboratory experiments [13]. Moreover, in most numerical simulations of dynamic rupture propagation with prescribed friction laws, $d_{c}$ is imposed a priori and its value is often assumed to be constant and uniform on the fault plane.

\subsection{The inverse problem}

Understanding the physics that controls the critical slip distance and explains the gap between observations from experimental and natural faults is one of the crucial problems in both the seismology and laboratory communities [14]. Furthermore, inferring the causality between fault activation and fault slip is important for seismic design and monitoring of underground structures [15], bridges [16], reinforced concrete buildings [17], carbon sequestration projects [18] and enhanced geothermal systems [19]. In lieu of that, an inverse problem is formulated with the fault slip acceleration being the input and critical slip distance being the output. We consider the relationship between acceleration $a_{i}(t)$ and the model response by the following statistical model

$$
a\left(t_{i}\right)=\mathcal{F}\left(t_{i}, d_{c}\right)+\epsilon_{i}
$$

where $\epsilon_{i}$ is the error in the statistical model. Here the $a\left(t_{i}\right)$ and $\epsilon_{i}$ are the random variables. The synthetic data over time $a\left(t_{1}\right), \ldots, a\left(t_{n}\right)$ are the $n$ observations for $a\left(t_{i}\right)$ and $\mathcal{F}\left(t_{i}, d_{c}\right)$ is the acceleration response of the model over time obtained using the forward model. The goal of the inverse problem is to determine the model parameter $d_{c}$ from the Eq. (2) using Bayesian inference [20] with Markov chain Monte Carlo (MCMC) [21-27] to generate samples from the posterior distribution.

\section{Bayesian Inversion and MCMC Framework}

The following components are present

$\checkmark$ data $D$

$\checkmark$ model M 
$\checkmark$ parameters $\boldsymbol{\theta}$

$\checkmark$ prior $P(\boldsymbol{\theta} \mid M)$

$\checkmark$ likelihood $P(\boldsymbol{D} \mid \boldsymbol{\theta})$

$\checkmark$ posterior $P(\boldsymbol{\theta} \mid \boldsymbol{D})$

A key part of Bayesian inversion is to express the posterior using Bayes' theorem [28] given by

$$
P(\boldsymbol{\theta} \mid \boldsymbol{D})=\frac{P(\boldsymbol{D} \mid \boldsymbol{\theta}) P(\boldsymbol{\theta} \mid M)}{\int P(\boldsymbol{D} \mid \boldsymbol{\theta}) P(\boldsymbol{\theta} \mid M) d \boldsymbol{\theta}}
$$

A tractable property of Bayesian inversion is that small changes in data lead to small changes in the posterior even when the inverse problem is ill-posed in the classical sense, so Bayesian inversion is stable. The posterior is however quite complicated with no closed form expression, so much of the contemporary research focuses on realizing the aforementioned advantages with Bayesian inversion without requiring access to the full posterior.

\subsection{Prior}

$P(\boldsymbol{\theta} \mid M)$ is often referred to as the prior. This describes the probability of having a particular set of values for our given model $\mathrm{M}$ before conditioning on our data. Because this is independent of the data, this term is often interpreted as representing our "prior beliefs" about what the parameterss should be based on previous measurements, physical concerns, and other known factors. In practice, this has the effect of essentially "augmenting" the data with other information.

\subsection{Likelihood}

$P(\boldsymbol{D} \mid \boldsymbol{\theta})$ is often referred to as the likelihood. The data likelihood is often given from insight into the physics of how data is generated (simulator).

\subsection{Posterior}

Finally, $P(\boldsymbol{\theta} \mid \boldsymbol{D})$ represents our posterior. This quantifies our belief in parameters after combining our prior intuition $P(\boldsymbol{\theta} \mid M)$ (prior) with current observations $P(\boldsymbol{D} \mid \boldsymbol{\theta})$ (likelihood). The posterior will be some compromise between the prior and the likelihood, with the exact combination depending on the strength and properties of the prior and the quality of the data used to derive the likelihood. 


\subsection{Markov chain Monte Carlo}

The calculation of these quantities reduces to the evaluation of the following integral

$$
\int h(\boldsymbol{\theta}) P(\boldsymbol{\theta} \mid \boldsymbol{D}) d \boldsymbol{\theta}
$$

under various choices of the function $h$. The idea behind MCMC is that one can produce a simulated sample $\left\{\boldsymbol{\theta}^{(1)}, \boldsymbol{\theta}^{(2)}, \ldots, \boldsymbol{\theta}^{(M)}\right\}$ from this posterior density leading to an evaluation of the integral:

$$
M^{-1} \sum_{j=1}^{M} h\left(\boldsymbol{\theta}^{(j)}\right) \stackrel{M \rightarrow \infty}{\longrightarrow} \int h(\boldsymbol{\theta}) P(\boldsymbol{\theta} \mid \boldsymbol{D}) d \boldsymbol{\theta}
$$

The formal definition of MCMC is that it is a method to sample a given multivariate distribution $\pi^{*}$ by construcing a suitable Markov chain with the property that its limiting, invariant distribution, is the target distribution $\pi^{*}$.

\subsubsection{Metropolis-Hastings algorithm}

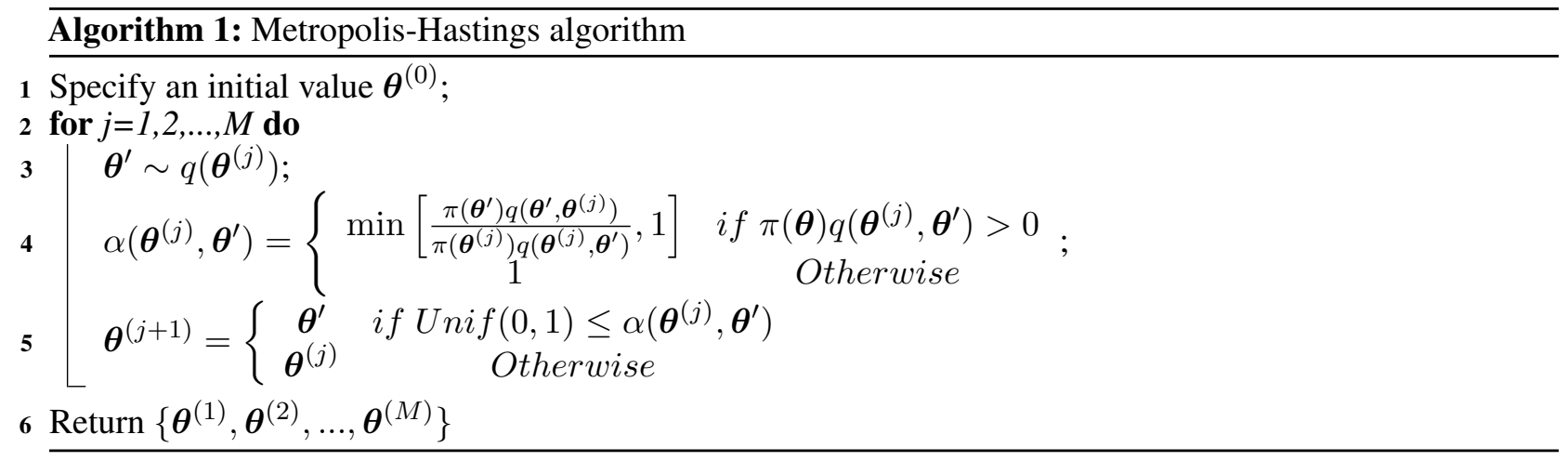

The Metropolis-Hastings (M-H) method is a general MCMC method to produce sample variates from a given multivariate density. As shown in Algorithm 1, it is based on a candidate generating density that is used to supply a proposal value and a probability of move that is used to determine if the proposal value should be taken as the next item of the chain. The probability of move is based on the ratio of the target density (evaluated at the proposal value in the numerator and the current value in the denominator) times the ratio of the proposal density (at the current value in the numerator and the proposal value in the denominator). The method is extremely general and powerful, it being possible in principle to view almost any MCMC algorithm, in one way or another, as a variant of the $\mathrm{M}-\mathrm{H}$ algorithm. 


\section{Bayesian applied to our problem}

We define the probability distribution of the critical slip distance, $d_{c}$, as a posterior distribution for the given acceleration data and the predictive model. The parametric posteriors distribution arrived from the Bayes theorem is as shown below

$$
\pi\left(d_{c} \mid a\left(t_{1}\right), \ldots, a\left(t_{n}\right)\right)=\frac{\pi\left(a\left(t_{1}\right), \ldots, a\left(t_{n}\right) \mid d_{c}\right) \pi_{0}\left(d_{c}\right)}{\int_{d_{c}} \pi\left(a\left(t_{1}\right), \ldots, a\left(t_{n}\right) \mid d_{c}\right) \pi_{0}\left(d_{c}\right) d d_{c}}
$$

Here $n$ is the number of data points, $\pi\left(d_{c} \mid a\left(t_{1}\right), \ldots, a\left(t_{n}\right)\right)$ is the posterior, $\pi\left(a\left(t_{1}\right), \ldots, a\left(t_{n}\right) \mid d_{c}\right)$ is the likelihood, and $\pi_{0}\left(d_{c}\right)$ is the prior distribution for the model parameters. Assuming the $\epsilon_{i} \sim N\left(0, \sigma^{2}\right)$ as unbiased, independent and identical normal distribution with standard deviation $\sigma$, the likelihood function is expressed as

$$
\pi\left(a\left(t_{1}\right), \ldots, a\left(t_{n}\right) \mid d_{c}\right)=\prod_{i=1}^{n} \pi\left(a\left(t_{i}\right) \mid d_{c}\right)=\prod_{i=1}^{n} \frac{1}{\sigma \sqrt{2 \pi}} e^{-\frac{1}{2}\left(\frac{a\left(t_{i}\right)-\mathcal{F}\left(t_{i}, d_{c}\right)}{\sigma}\right)^{2}}
$$

The data points $a\left(t_{1}\right), \ldots, a\left(t_{n}\right)$ in Eq. (5) are generated from the forward model explained next

\subsection{The forward model}



Figure 1: Spring slider damper idealization of fault activation

Firstly, the RSF model Eq. (1) is rewritten as

$$
V=V_{0} \exp \left(\frac{1}{A}\left(\mu-\mu_{0}-B \ln \left(\frac{V_{0} \theta}{d_{c}}\right)\right)\right)
$$

to obtain $\dot{V}$ as

$$
\dot{V}=\frac{V}{A}\left(\dot{\mu}-\frac{B}{\theta} \dot{\theta}\right)
$$


To obtain $\dot{\mu}$, we deploy the spring slider damper model as shown in Fig. 1. The stiffness $k$ represents elastic interactions between the fault patch and the ductile deeper part of the fault, and is a function of the fault length $l$ and elastic modulus $E$ as $k \approx \frac{E}{l}$. This simple model assumes that slip, stress, and friction law parameters are uniform on the fault patch. The friction coefficient of the block is given by

$$
\mu=\frac{\tau}{\sigma}=\frac{\tau_{l}-k \delta-\eta V}{\sigma}
$$

where $\sigma$ is the normal stress, $\tau$ the shear stress on the interface, $\tau_{l}$ is the remotely applied stress acting on the fault in the absence of slip, $-k \delta$ is the decrease in stress due to fault slip [1] and $\eta$ is the radiation damping coefficient [29]. We consider the case of a constant stressing rate $\dot{\tau}_{l}=k V_{l}$ where $V_{l}$ is the load point velocity. In lieu of Eq. (8), we obtain the time derivative of the friction coefficient as

$$
\dot{\mu}=k^{\prime}\left(V_{l}-V\right)-k^{\prime \prime} \dot{V}
$$

where $k^{\prime}=\frac{E}{l \sigma}$ and $k^{\prime \prime}=\frac{\eta}{\sigma}$. From Eqs. (1), (7) and (9), we get

$$
\dot{V}=\left(\frac{A}{V}+k^{\prime \prime}\right)^{-1}\left(k^{\prime}\left(V_{l}-V\right)-\frac{B}{\theta}\left(1-\frac{\theta V}{d_{c}}\right)\right)
$$

In lieu of Eqs. (6) and (9), the forward model is written as

$$
\begin{aligned}
V & =V_{0} \exp \left(\frac{1}{A}\left(\mu-\mu_{0}-B \ln \left(\frac{V_{0} \theta}{d_{c}}\right)\right)\right) \\
\mathcal{F}\left(t, d_{c}\right) & =\left(\frac{A}{V}+k^{\prime \prime}\right)^{-1}\left(k^{\prime}\left(V_{l}-V\right)-\frac{B}{\theta}\left(1-\frac{\theta V}{d_{c}}\right)\right)
\end{aligned}
$$

\subsection{System response for ballpark values}

We employ the load point velocity variation of the form

$$
V_{l}=V_{\text {ref }}(1+\exp (-t / 10) \sin (10 t))
$$

The ballpark values are taken from [1] and [29] as follows: elastic modulus $E=$ $5 \times 10^{10} \mathrm{~Pa}$, critical fault length $l=3 \times 10^{-2} \mathrm{~m}$, normal stress $\sigma=200 \times 10^{6} \mathrm{~Pa}$, radiation damping coefficient $\eta=20 \times 10^{6} \mathrm{~Pa} /(\mathrm{m} / \mathrm{s}), A=0.011$ and $B=0.014$. The effective stiffness and damping is obtained as $k^{\prime} \approx 10^{-2}[1 / \mu \mathrm{m}]$ and $k^{\prime \prime}=$ $10^{-7}[\mathrm{~s} / \mu \mathrm{m}]$ respectively. The reference values are $V_{\text {ref }}=1 \mu \mathrm{m} / \mathrm{s}, \theta_{0}=0.6$ and $\mu_{\text {ref }}=\mu_{0}=0.6$. As shown in Appendix A, we employ SciPy [30] to arrive at acceleration time series, which is plotted for critical slip distance parameter values ranging over three orders of magnitude in Fig. 2. 

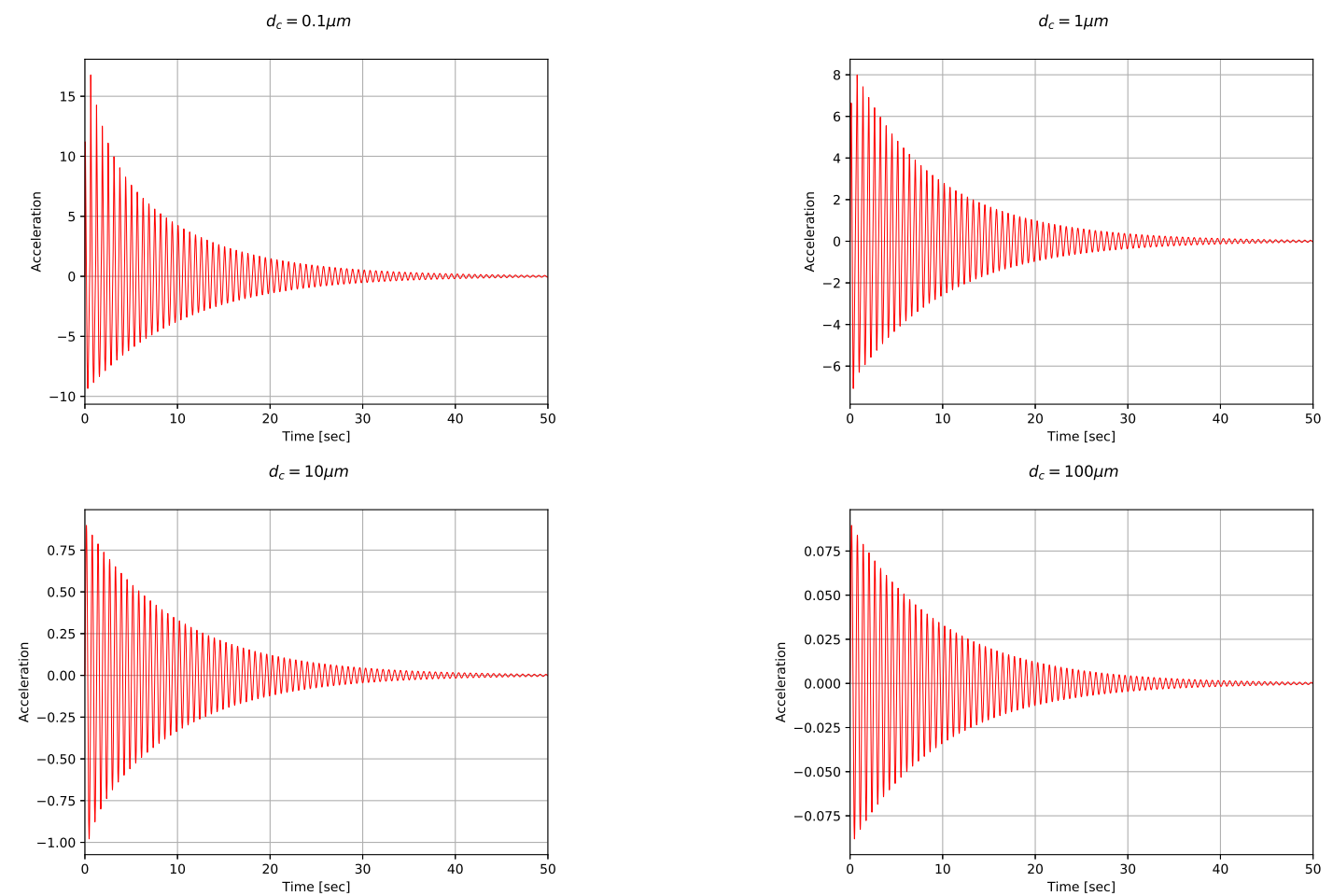

Figure 2: System response for different values of critical slip distance over three orders of magnitude. Units of acceleration are $\mu \mathrm{m} / \mathrm{s}^{2}$

\section{Conclusions and Outlook}

We presented a Bayesian inference and MCMC based framework to arrive at a probability distribution of the critical slip distance parameter in the RSF model with the fault slip acceleration as the input to the inverse problem. We deployed the popularly used SSD model to generate data sets of the fault slip acceleration. While the solution of inversion problem with the fault slip acceleration as the input and a parameter of RSF model is a step forward, the eventual goal is the solution of an inverse problem with the seismograph reading of the earthquake as the input and parameters of the appropriate forward model as the output. It can be argued that the fault slip acceleration is of the same order of magnitude as the seismograph reading, but such an abstraction would be a gross over-simplification of the problem at hand. The slipping fault generates a stress field which spawns off seismic waves which then reach the seismograph typically mounted on the earth's surface. Any forward model which encompasses the domain going all the way to the earth's surface [31] and considers the impact of pore pressure perturbations on fault activation [32] would be more appropriate for the subsequent inverse problem. The computational framework popularly used to study the impact of pore 
pressure perturbations on geotechnics is the fixed stress split staggered solution algorithm [31-40]. Furthermore, the effect of wave propagation needs to be considered for faults slipping at moderate to high speeds to accurately capture the induced seismicity. A two-way coupled poroelastodynamics forward model in the presence of faults with the parameters identified for a subsequent inversion using Bayesian and MCMC with the seismograph readin as the input is the way forward. Be that as it may, it is important to identify the problem statement at hand without necessarily resorting to computational overkill.

\section{A Python code to generate acceleration time series}

def friction $(\mathrm{t}, \mathrm{y})$ : \# $y[0]$ is mu, y[1] is theta, y[2] is velocity dydt $=n p \cdot z \operatorname{eros}((\operatorname{len}(y), 1))$

power $=1 / \mathrm{a} *\left(\mathrm{y}[0]-\mathrm{mu} \_\right.$ref $-\mathrm{b} * \log \left(\mathrm{V} \_\right.$ref $\left.\left.* \mathrm{y}[1] / \mathrm{Dc}\right)\right)$

$\mathrm{v}=\mathrm{V}_{-}$ref $* \exp ($ power $) \#$ Compute $\mathrm{v}$

dydt $[1]=1 .-\mathrm{v} * \mathrm{y}[1] / \mathrm{Dc} \#$ Compute time derivative

of theta

dydt $[0]=\operatorname{kprime} * \mathrm{~V}_{-} \_-\operatorname{kprime} * \mathrm{~V}$

$\mathrm{dydt}[2]=\mathrm{v} / \mathrm{a} *(\mathrm{dydt}[0]-\mathrm{b} / \mathrm{y}[1] * \mathrm{dydt}[1])$

dydt $[0]=\operatorname{dydt}[0]-\mathrm{k} 1 * \mathrm{dydt}[2]$ \# Compute time

derivative of mu

dydt [2] $=\mathrm{v} / \mathrm{a} *(\mathrm{dydt}[0]-\mathrm{b} / \mathrm{y}[1] * \mathrm{dydt}[1]) \#$ Compute

time derivative of velocity

return dydt

$r=$ integrate.ode(friction). set_integrator ('vode', order $=5$, max_step $=0.001$, method $=$ ' bdf', atol $=1 \mathrm{e}-10$, rtol $=1 \mathrm{e}-6)$ \# Use integrate functionality within Python package SciPy

r.set_initial_value ([0.6,Dc/V_ref, V_ref $], t_{-}$start) \# Initial conditions on mu, theta, velocity

$\mathrm{k}=1$

while r.successful () and $\mathrm{k}<$ num_steps:

r. integrate $\left(\mathrm{r} . \mathrm{t}+\mathrm{delta}{ }_{-} \mathrm{t}\right)$ \# Integrate the $\operatorname{ODE}(\mathrm{s})$ across each delta_t timestep [2]

$\mathrm{t}[\mathrm{k}], \mathrm{mu}[\mathrm{k}], \mathrm{theta}[\mathrm{k}], \mathrm{vel}[\mathrm{k}]=\mathrm{r} \cdot \mathrm{t}, \mathrm{r} \cdot \mathrm{y}[0], \mathrm{r} \cdot \mathrm{y}[1], \mathrm{r} \cdot \mathrm{y}$ 


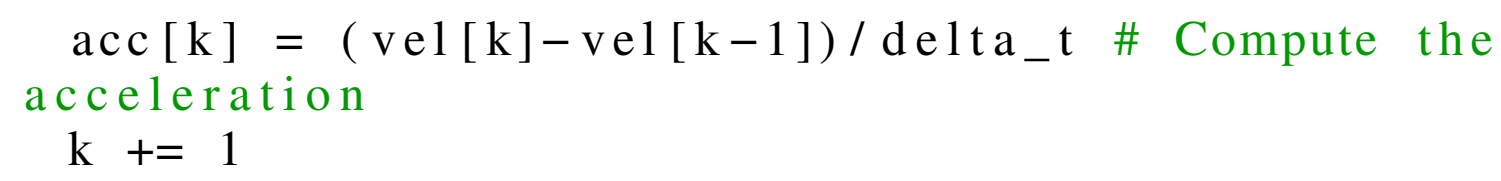

Listing 1: Python code for forward response of spring slider damper idealization

\section{References}

[1] Hiroo Kanamori and Emily E Brodsky. The physics of earthquakes. Reports on Progress in Physics, 67(8):1429, 2004.

[2] Christopher H Scholz, Yen Joe Tan, and Fabien Albino. The mechanism of tidal triggering of earthquakes at mid-ocean ridges. Nature communications, 10(1):1-7, 2019.

[3] Sebastian Hainzl, Toni Kraft, Joachim Wassermann, Heiner Igel, and E Schmedes. Evidence for rainfall-triggered earthquake activity. Geophysical Research Letters, 33(19), 2006.

[4] EK Montgomery-Brown, David R Shelly, and Paul A Hsieh. Snowmelttriggered earthquake swarms at the margin of long valley caldera, california. Geophysical Research Letters, 46(7):3698-3705, 2019.

[5] ChiChing Liu, Alan T Linde, and I Selwyn Sacks. Slow earthquakes triggered by typhoons. Nature, 459(7248):833-836, 2009.

[6] Gillian R Foulger, Miles P Wilson, Jon G Gluyas, Bruce R Julian, and Richard J Davies. Global review of human-induced earthquakes. EarthScience Reviews, 178:438-514, 2018.

[7] A. L. Ruina. Slip instability and state variable friction laws. Geophys. Res. Lett., 88:359-370, 1983.

[8] C. H. Scholz. Mechanics of faulting. Ann. Rev. Earth Planet. Sci., 17:309334, 1989.

[9] C. Marone. Laboratory-derived friction laws and their application to seismic faulting. Ann. Rev. Earth Planet. Sci., 26:643-696, 1998.

[10] Andrew Clennel Palmer and James Robert Rice. The growth of slip surfaces in the progressive failure of over-consolidated clay. Proceedings of the Royal Society of London. A. Mathematical and Physical Sciences, 332(1591):527548, 1973.

[11] Christopher H Scholz. The mechanics of earthquakes and faulting. Cambridge university press, 2019. 
[12] Yoshihiro Kaneko, Eiichi Fukuyama, and Ian James Hamling. Slipweakening distance and energy budget inferred from near-fault ground deformation during the 2016 mw7. 8 kaikōura earthquake. Geophysical Research Letters, 44(10):4765-4773, 2017.

[13] André Niemeijer, Giulio Di Toro, Stefan Nielsen, and Fabio Di Felice. Frictional melting of gabbro under extreme experimental conditions of normal stress, acceleration, and sliding velocity. Journal of Geophysical Research: Solid Earth, 116(B7), 2011.

[14] Mitiyasu Ohnaka. A constitutive scaling law and a unified comprehension for frictional slip failure, shear fracture of intact rock, and earthquake rupture. Journal of Geophysical Research: Solid Earth, 108(B2), 2003.

[15] Youssef MA Hashash, Jeffrey J Hook, Birger Schmidt, I John, and Chiang Yao. Seismic design and analysis of underground structures. Tunnelling and underground space technology, 16(4):247-293, 2001.

[16] MJ Nigel Priestley, Frieder Seible, and Gian Michele Calvi. Seismic design and retrofit of bridges. John Wiley \& Sons, 1996.

[17] Jack Moehle. Seismic design of reinforced concrete buildings. McGraw-Hill Education, 2015.

[18] Mark D Zoback and Steven M Gorelick. Earthquake triggering and largescale geologic storage of carbon dioxide. Proceedings of the National Academy of Sciences, 109(26):10164-10168, 2012.

[19] William L Ellsworth, Domenico Giardini, John Townend, Shemin Ge, and Toshihiko Shimamoto. Triggering of the pohang, korea, earthquake $(\mathrm{m} \mathrm{w}$ 5.5) by enhanced geothermal system stimulation. Seismological Research Letters, 90(5):1844-1858, 2019.

[20] PL Green and K Worden. Bayesian and markov chain monte carlo methods for identifying nonlinear systems in the presence of uncertainty. Philosophical Transactions of the Royal Society A: Mathematical, Physical and Engineering Sciences, 373(2051):20140405, 2015.

[21] Klaus Mosegaard and Albert Tarantola. Monte carlo sampling of solutions to inverse problems. Journal of Geophysical Research: Solid Earth, 100(B7):12431-12447, 1995.

[22] Joshua S Speagle. A conceptual introduction to markov chain monte carlo methods. arXiv preprint arXiv:1909.12313, 2019. 
[23] Karthik Reddy Lyathakula and Fuh-Gwo Yuan. A probabilistic fatigue life prediction for adhesively bonded joints via anns-based hybrid model. International Journal of Fatigue, page 106352, 2021.

[24] Karthik Reddy Lyathakula and Fuh-Gwo Yuan. Probabilistic fatigue life prediction for adhesively bonded joints via surrogate model. In Sensors and Smart Structures Technologies for Civil, Mechanical, and Aerospace Systems 2021, volume 11591, page 115910S. International Society for Optics and Photonics, 2021.

[25] Karthik Reddy Lyathakula and Fuh-Gwo Yuan. Fatigue damage prognosis of adhesively bonded joints via a surrogate model. In Sensors and Smart Structures Technologies for Civil, Mechanical, and Aerospace Systems 2021, volume 11591, page 115910K. International Society for Optics and Photonics, 2021.

[26] Stephen Brooks. Markov chain monte carlo method and its application. Journal of the royal statistical society: series D (the Statistician), 47(1):69-100, 1998.

[27] Siddhartha Chib. Markov chain monte carlo methods: computation and inference. Handbook of econometrics, 5:3569-3649, 2001.

[28] Thomas Bayes. Lii. an essay towards solving a problem in the doctrine of chances. by the late rev. mr. bayes, frs communicated by mr. price, in a letter to john canton, amfr s. Philosophical transactions of the Royal Society of London, (53):370-418, 1763.

[29] Mark W McClure and Roland N Horne. Investigation of injection-induced seismicity using a coupled fluid flow and rate/state friction model. Geophysics, 76(6):WC181-WC198, 2011.

[30] Pauli Virtanen, Ralf Gommers, Travis E Oliphant, Matt Haberland, Tyler Reddy, David Cournapeau, Evgeni Burovski, Pearu Peterson, Warren Weckesser, Jonathan Bright, et al. Scipy 1.0: fundamental algorithms for scientific computing in python. Nature methods, 17(3):261-272, 2020.

[31] Saumik Dana, Xiaoxi Zhao, and Birendra Jha. A two-grid computational framework for fast monitoring of fault stability and ground deformation in multiphase geomechanics. Under review at Journal of Computational Physics, 2021.

[32] Saumik Dana and Birendra Jha. A fault slip model to study earthquakes due to pore pressure perturbations. arXiv preprint arXiv:2104.06257, 2021. 
[33] Saumik Dana and Mary F Wheeler. Design of convergence criterion for fixed stress split iterative scheme for small strain anisotropic poroelastoplasticity coupled with single phase flow. arXiv preprint arXiv:1912.06476, 2019.

[34] Saumik Dana. System of equations and staggered solution algorithm for immiscible two-phase flow coupled with linear poromechanics. arXiv preprint arXiv:1912.04703, 2019.

[35] Saumik Dana, Joel Ita, and Mary F Wheeler. The correspondence between voigt and reuss bounds and the decoupling constraint in a two-grid staggered algorithm for consolidation in heterogeneous porous media. Multiscale Modeling \& Simulation, 18(1):221-239, 2020.

[36] Saumik Dana, Xiaoxi Zhao, and Birendra Jha. Two-grid method on unstructured tetrahedra: Applying computational geometry to staggered solution of coupled flow and mechanics problems. arXiv preprint arXiv:2102.04455, 2021.

[37] S. Dana and M. F. Wheeler. Convergence analysis of fixed stress split iterative scheme for anisotropic poroelasticity with tensor biot parameter. Computational Geosciences, 22(5):1219-1230, 2018.

[38] S. Dana and M. F. Wheeler. Convergence analysis of two-grid fixed stress split iterative scheme for coupled flow and deformation in heterogeneous poroelastic media. Computer Methods in Applied Mechanics and Engineering, 341:788-806, 2018.

[39] Saumik Dana, Benjamin Ganis, and Mary F. Wheeler. A multiscale fixed stress split iterative scheme for coupled flow and poromechanics in deep subsurface reservoirs. Journal of Computational Physics, 352:1-22, 2018.

[40] S. Dana. Addressing challenges in modeling of coupled flow and poromechanics in deep subsurface reservoirs. PhD thesis, The University of Texas at Austin, 2018. 